\title{
In vivo therapeutic evaluation of polymeric nanomedicines: effect of different targeting peptides on therapeutic efficacy against breast cancer
}

\author{
Yongmei Zhao, Nicholas L. Fletcher, Tianqing Liu, Anna C. Gemmell, Zachary H. Houston, Idriss Blakey \\ and Kristofer J. Thurecht ${ }^{\bowtie}$ \\ Centre for Advanced Imaging, Australian Institute for Bioengineering and Nanotechnology and ARC Centre of Excellence in Convergent Bio-Nano Science and \\ Technology, University of Queensland, Brisbane, 4072, Australia \\ QIMR Berghofer Medical Research Institute, 300 Herston Road, Brisbane, QLD, 4006, Australia \\ $\bowtie$ Corresponding author: k.thurecht@uq.edu.au \\ (C) Ivyspring International Publisher. This is an open access article distributed under the terms of the Creative Commons Attribution (CC BY-NC) license \\ (https://creativecommons.org/licenses/by-nc/4.0/). See http://ivyspring.com/terms for full terms and conditions.
}

Received: 2018.05.11; Accepted: 2018.08.12; Published: 2018.08.24

\begin{abstract}
Targeted nanomedicines offer many advantages over macromolecular therapeutics that rely only on passive accumulation within the tumour environment. The aim of this work was to investigate the in vivo anticancer efficiency of polymeric nanomedicines that were conjugated with peptide aptamers that show high affinity for receptors on many cancer cells. In order to assess the ability for the nanomedicine to treat cancer and investigate how structure affected the behavior of the nanomedicine, three imaging modalities were utilized, including in vivo optical imaging, multispectral optoacoustic tomography (MSOT) and ex vivo confocal microscopy. An 8-mer (A8) or 13-mer (A13) peptide aptamer that have been shown to exhibit high affinity for heat shock protein 70 (HSP70) was covalently-bound to hyperbranched polymer (HBP) nanoparticles with the purpose of both cellular targeting, as well as the potential to impart some level of chemo-sensitization to the cells. Furthermore, doxorubicin was bound to the polymeric carrier as the anticancer drug, and Cyanine-5.5 (Cy5.5) was incorporated into the polymer as a monomeric fluorophore to aid in monitoring the behavior of the nanomedicine. Enhanced tumour regression was observed in nude mice bearing MDA-MB-468 xenografts when the nanocarriers were targeted using the peptide ligands, compared to control groups treated with free DOX or HBP without aptamer. The accumulated DOX level in solid tumours was 5.5 times higher in mice treated with the targeted therapeutic, than mice treated with free DOX, and 2.6 times higher than the untargeted nanomedicine that relied only on passive accumulation. The results suggest that aptamer-targeted therapeutics have great potential for improving accumulation of nanomedicines in tumours for therapy.
\end{abstract}

Key words: peptide aptamers, chemo-sensitization, Multispectral Optoacoustic Tomography (MSOT), nanomedicine

\section{Introduction}

Breast cancer, which involves the abnormal growth of cells lining the breast lobules or ducts, is the most frequently diagnosed cancer and a leading cause of cancer-related deaths in women world-wide.[1] At present, small molecules such as doxorubicin and other chemotherapeutic drugs are used as first-line treatments for the disease. However, due to poor tissue selectivity, these chemotherapeutic drugs consistently result in serious side effects, such as cardiotoxicity caused by doxorubicin.[2-3] It has also been observed that during the course of treatment, many tumours develop multidrug resistance (MDR) which is a major impediment to current cancer therapies.[4] In order to address these problems, nanoparticles have been widely investigated as an alternative formulation for drug delivery in cancer treatment.[5],[6] One particular variety of multifunctional nanoparticles combining therapeutic, diagnostic, and sensing modalities in one nanostructure, a so-called theranostic, is a promising concept in cancer therapy.[7-12]

Stress-inducible heat shock protein 70 (HSP70) 
assisting in protein stabilization and translocation of polypeptides across the cell membrane is indispensable for cell survival under conditions of stress.[13] It has been found to be over-expressed in many types of cancers including breast cancer.[14-17] Therefore, targeting and potentially inhibiting HSP70 is a promising strategy in cancer therapy.[18] Rérole and coworkers have investigated a library of small peptide-aptamers and found a number of potential candidates that exhibited high affinity for HSP70, as well as enhanced chemo-sensitization.[19] Thus, these aptamers show significant potential as a therapeutic strategy for overcoming multidrug resistance.

From our previous work, we successfully developed a hyperbranched polymeric theranostic nanoparticle bearing an A8 aptamer (as defined by

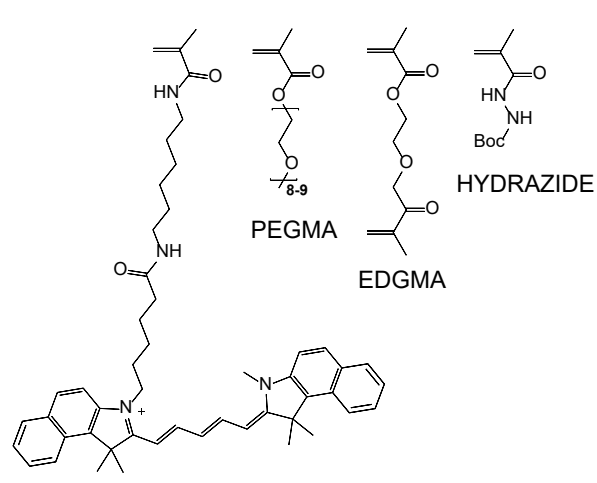

Cy5.5-methacrylamide

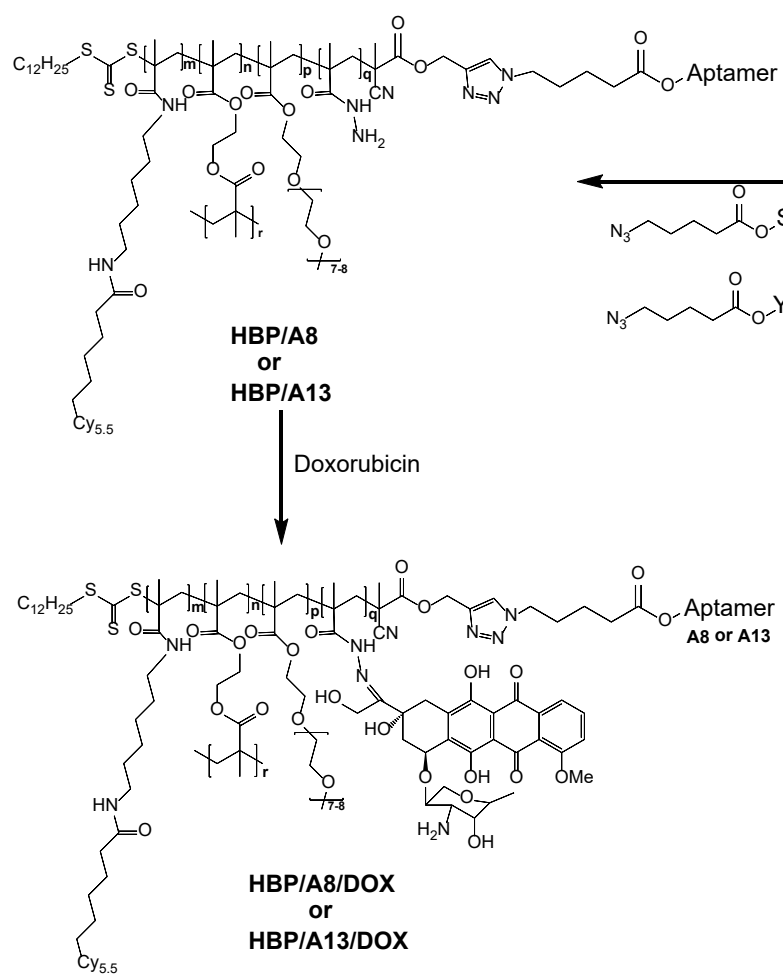

Rérole et al. [19]) as a targeting moiety, DOX as chemotherapeutic agent via a $\mathrm{pH}$ degradable hydrazone bond and a fluorescent dye (Cy-5) as a diagnostic and imaging agent.[20], [21] This study also demonstrated enhanced accumulation of polymers bearing an 8-mer aptamer within solid tumours in mice compared to the untargeted analogue. However, no studies have demonstrated the effect of these aptamers on the therapeutic efficacy of the nanoparticles. Motivated by the promising enhancement in accumulation of the nanomedicines in tumour tissue, here we investigate the therapeutic efficiency of this polymeric theranostic delivery system for two different peptide aptamers as potential targeting ligands, A8 and A13 (Figure 1).

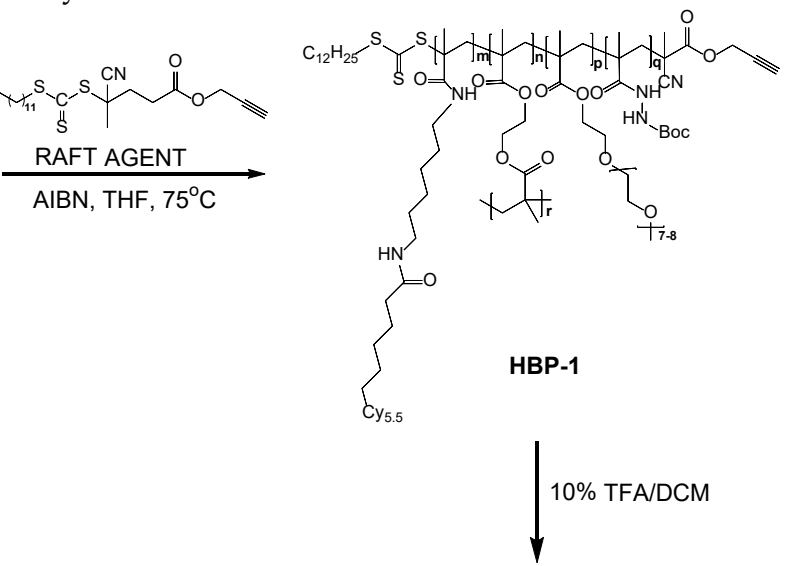

SPWPRPTY

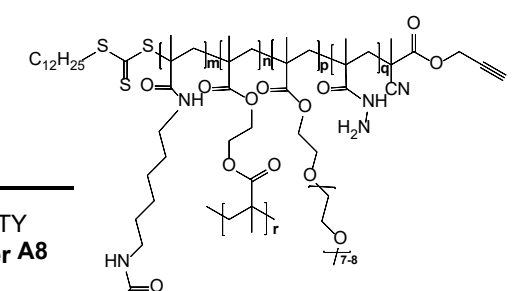

ACAYYSPRHKTTF
Aptamer A13

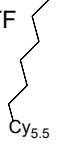

HBP-2
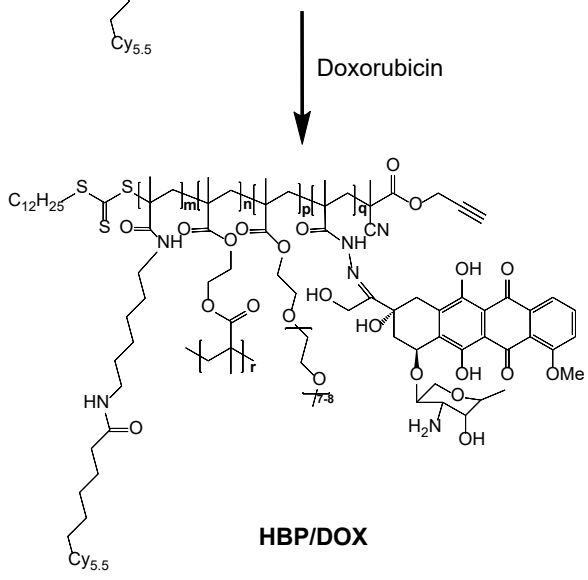

Figure 1. The synthetic pathway for development of the hyperbranched polymer based theranostic nanomedicine incorporating either $\mathrm{A} 8$ (HBP/A8/DOX) or A13 (HBP/A13/DOX). 
The main focus was to assess whether the two peptides exhibited improved targeting of nanomedicines leading to enhanced therapeutic efficacy, while minimizing undesirable side-effects; such an assessment not only looked at the tumour regression, but also on the ability for the nanomedicine and/or drug molecules to diffuse through tumour tissue. A secondary motivator was to assess whether one peptide was more efficient than the other at increasing tissue accumulation when attached to the nanomedicine, since Rérole et al.[19] showed varying efficacy for these two peptides. We were able to independently track the biodistribution and intracellular distribution of both the DOX and the hyperbranched polymer nanocarrier during in vivo analysis using optical imaging and multispectral optoacoustic tomography. This was achieved by using the native fluorescence of DOX and functionalizing the hyperbranched polymer with a fluorescent dye (Cy-5.5). Finally, a tumour regression study was carried out in nude mice bearing MDA-MB-468 xenografts to determine the efficacy of the targeted therapeutic for treating breast cancers.

\section{Methods}

\section{Materials}

Modified peptide sequences: $\mathrm{N}$ - terminal (5-azidopentanoic acid)- YCAYYSPRHKTTF and Nterminal (5-azidopentanoic acid)-SPWPRPTY were synthesised at the Australian Biobest Biotechnology Service. Doxorubicin hydrochloride, trifluoroacetic acid (TFA), dicyclohexylcarbodiimide (DCC), methacryloyl chloride, tert-butyl carbazate, 4-dimethylaminopyridine (DMAP), 1-ethyl-3-(3dimethylaminopropyl) carbodiimide hydrochloride (EDC $\cdot \mathrm{HCl})$, and 4,4'-azobis(4-cyanopentanoic acid) were bought from Sigma Aldrich and used directly without any purification. Cyanine-5.5 amine was purchased from Lumiprobe. Azobis(isobutyronitrile) (AIBN; Sigma Aldrich) was recrystallized twice from methanol before use. Solvents including n-hexane, ethyl acetate, dichloromethane (DCM), dimethylformamide (DMF), diethyl ether, pyridine, tetrahydrofuran, acetonitrile and methanol were used dry where applicable and of reagent grade quality. Poly(ethylene glycol methacrylate) (PEGMA, MW = $475 \mathrm{~g} \mathrm{~mol}^{-1}$ ) and, ethylene glycol dimethacrylate (EGDMA) were purified to remove radical inhibitors before use by passing through a basic alumina column. Ultrapure water $\left(18.2 \mathrm{M} \Omega . \mathrm{cm}\right.$ at $\left.25^{\circ} \mathrm{C}\right)$ was obtained from an Elga ultra-pure water system.

\section{Cell culture}

Trypsin, trypan blue solution, and phosphate buffered saline (PBS) were purchased from
ThermoFisher Scientific. MDA-MB-468 breast cancer cells were incubated at $37{ }^{\circ} \mathrm{C}$ in a humidified atmosphere of $5 \% \mathrm{CO}_{2}$ in air. Cells were maintained in RPMI medium (Gibco) supplemented with $10 \%$ (v/v) foetal bovine serum (heat inactivated, Bovogen), 100 $\mathrm{U} / \mathrm{mL}$ Penicillin, $100 \mu \mathrm{g} / \mathrm{mL}$ Streptomycin and $2 \mathrm{mM}$ L-glutamine (Gibco).

\section{Synthesis of nanomaterials}

The synthetic pathway and detailed synthesis procedure for hyperbranched polymers was described in our previous work,[22, 23] and all subsequent characterization including ${ }^{1} \mathrm{H}$ NMR and $\mathrm{UV} /$ Vis for the polymers used in this publication are listed in a previous publication.[20] For detection purposes, there was one Cy5.5 molecule per ten HBP molecules.

\section{Characterisation of polymeric nanomedicine}

${ }^{1} \mathrm{H}$ NMR spectra were acquired on a Bruker Avance 400 spectrometer. Size distribution and zeta potential were measured by Dynamic Light Scatting (DLS) at $25^{\circ} \mathrm{C}$. Gel Permeation Chromatography Multiangle Laser Light Scattering (GPC-MALLS) with THF as an eluent at a flow rate of $1 \mathrm{~mL} / \mathrm{min}$ was used to measure the molecular weight and dispersity of the polymers.

UV-Vis and fluorescence spectra (Tecan M200 Infinite pro plate reader) of free DOX and DOX incorporated into a representative nanoformulation are provided in Figure S5 $\left(25^{\circ} \mathrm{C}\right.$, PBS), showing minimal change in spectral properties for the DOX under both conditions.

\section{In vivo anti-tumour efficacy study}

All studies were in accordance with guidelines of the Animal Ethics Committee of The University of Queensland, and the Australia Code for the Care and Use of Animals for Science Purposes. Mice were anesthetized with $2 \%$ isoflurane in oxygen at a flow rate of $2 \mathrm{~mL} / \mathrm{min}$. BALB/c nude mice (Australian Resources Centre, Australia) were used to establish human breast cancer xenografts by injection of $5 \times 10^{6}$ MDA-MB-468 cells into the left inguinal mammary line and tumour growth was monitored by calliper measurements twice per week.

After 2 weeks of cell growth when the tumours had reached $\sim 100 \mathrm{~mm}^{3}$, in vivo tumour regression studies were performed. Six groups were used in the study: vehicle control (saline), free DOX, HBP/DOX, HBP/A8/DOX, HBP/A13/DOX, HBP/A13 co-administered with free DOX. In each case, the formulations were injected through the tail vein using a $29 \mathrm{G}$ needle. To enable comparison of the different formulations, with the exception of the saline control, each injection contained a dose of $4.5 \mathrm{mg}$ DOX / $\mathrm{kg}$ of 
mouse. Each treatment group consisted of 3 mice which were administered twice per week for 4 weeks; 8 injections in total. The tumour sizes were measured using an electronic digital caliper, and the mouse body weight was monitored throughout the experiment. At the conclusion of the experiments, mice were humanely sacrificed on day 30 and the tumours were collected for tissue sectioning. The tumour volume was calculated using the formula: tumour volume $=0.5 \times$ length $\times$ width $^{2}$. The cohort number $(n=3)$ was chosen based on power calculations to achieve statistical significance in tumour regression based on previous studies [12] and this is reflected in the significance levels present between treatments in Figure 2.

Ex vivo fluorescence images were acquired using a Carestream In-Vivo MS FX Pro (Bruker) imaging station with an excitation filter of $630 \mathrm{~nm}$ and an emission filter of $700 \mathrm{~nm}$ for Cy5.5 $(190 \mathrm{~mm}$ field-of-view, f-stop 2.8, $30 \mathrm{~s}$ acquisition time) and were co-registered with an X-ray image $(0.2 \mathrm{~mm}$ aluminium filter, $1.2 \mathrm{~s}$ acquisition time). Live animal fluorescence imaging was performed at 2 day intervals after injecting each drug formulation into the tail vein of each group of mice to monitor nanomedicine distribution.

\section{In vivo multispectral optoacoustic tomography (MSOT)}

For optoacoustic imaging, an MSOT inVision 256-TF small animal imaging system (iThera Medical, Munich, Germany) was used.[24] The mice were horizontally positioned in a dedicated holder (iThera Medical) under 2\% isoflurane anaesthetic and wrapped in a thin polyethylene membrane with ultrasound coupling gel applied to provide contact between the animal and the membrane of the animal holder. The mouse was z-translated through the imaging plane in $0.5-\mathrm{mm}$ oversampling steps using a linear stage control to acquire a stack of $2 \mathrm{D}$ axial images over the tumour region which in turn allowed for optimal three-dimensional (3D) rendering. 10 frames at each of the following wavelengths were acquired in between wavelength-tuning at each position in all experiments in this study: $680,715,730$, $760,765,770,800$ and $845 \mathrm{~nm}$.

The images were reconstructed using a model-based algorithm and processed using linear spectral unmixing to identify the signal of Cy5.5 from other intrinsic photoabsorbers, oxy- and deoxygenated haemoglobin.[25] All in vivo MSOT images presented in the study were scaled to the same threshold (Cy5.5: 0-0.15 arbitrary units [au]; oxy- and deoxygenated hemoglobin: 0-20.0 au and 0-30.0 au respectively; background shown at $845 \mathrm{~nm}$ ) to enable visual comparison of signal intensities throughout. After 3D rendering of the acquired stack of 2D images, the mean MSOT signal intensities for Cy5.5 in the subcutaneous tumour were assessed by setting volumes of interest (VOI) around each of the tumours at their largest diameter using the ITK-SNAP software.[26]

\section{Ex vivo intratumoural distribution studies (Immunofluorescence)}

Tumours were embedded in Tissue-Tek OCT compound, snap frozen in liquid nitrogen and cut into $7 \mu \mathrm{m}$ thick frozen sections on glass slides using a cryostat. Sections were further prepared with routine immunofluorescent methods. Antibodies against CD31 (rabbit as host species) were used to stain the blood vessels (detection by goat anti-rabbit secondary antibody labelled with Alexa568; ex: 568 nm; em: 500-700 nm). After antibody incubation, sections were washed and counter-stained with DAPI. The slides were mounted with ProLong Gold and imaged with Zeiss confocal microscope using 20× magnification. The DOX distribution was acquired using a $488 \mathrm{~nm}$ excitation filter and a 500-700 nm emission filter.

\section{Histology studies}

After the treatment, mice from each group were humanely sacrificed and the tumours were collected and preserved for routine histology by fixation with $10 \%$ formalin. The fixed tumours were embedded in paraffin, sectioned to $5 \mu \mathrm{m}$, and stained with hematoxylin and eosin (H\&E) for tissue histological analysis.

\section{Statistical analysis}

All experimental data were obtained in triplicate unless otherwise mentioned and are presented as the mean \pm standard deviation. Statistical comparison by analysis of variance was performed using a Student's $t$-test and significance levels were reported in the text for each analysis.

\section{Results}

\section{Characterization of the two targeted nanomedicines: HBPIA8/DOX or HBPIA13/DOX in buffer}

The targeted nanomaterials were synthesised using a previously published procedure and all characterisation data is presented in a previous publication [20]. The targeting moieties (A13 and A8; structure shown in Figure 1) were successfully conjugated to the polymer using the copper catalysed 1,3-dipolar cycloaddition click reaction which was validated by complete disappearance of the proton resonance at $4.5 \mathrm{ppm}$ from ${ }^{1} \mathrm{H}$ NMR (attributed to the 
methylene resonance adjacent to the alkyne group at the polymer chain end). This peak was attributed to the methylene protons adjacent to the alkyne group in the precursor polymer and its disappearance indicated complete cyclisation to yield the product leading to $\sim 3.5$ aptamers per HBP. This confirmed the click reaction proceeded to high conversion and indicated that the chosen aptamer was successfully attached to each arm of the HBP (Supporting information S1). DOX was conjugated to the polymer using an acid-labile hydrazone bond in which there were approximately six DOX molecules per polymer as verified by UV-Vis spectroscopy. The drug was shown to release over 24 hours under conditions that replicate the $\mathrm{pH}$ of endosomes.[20] The hydrodynamic radii of the different materials were measured using diffusion coefficients calculated from ${ }^{1} \mathrm{H}$ NMR and correlated to the Stokes-Einstein equation which is widely used to determine hydrodynamic radii of polymeric particles in solution.[27], [28] Finally, the molecular weight was measured by GPS-MALLS (Table 1). All nanomedicines had hydrodynamic radii between 7-9 $\mathrm{nm}$. There was no significant change in size between HBPs conjugated with A8 or A13.

Table 1 Physico-chemical properties of polymeric nanomedicine

\begin{tabular}{lll}
\hline & $\mathrm{M}_{\mathrm{n}}$ SEC-MALLS $(\mathrm{kDa})$ & $\mathrm{D}_{\mathrm{h}^{\mathrm{a}}}(\mathrm{nm})$ \\
\hline HBP/DOX & ${ }^{\mathrm{b}}$ & $8 \pm 2$ \\
HBP/A13 & 34.0 & $9 \pm 2$ \\
HBP/A8 & 40.2 & $9 \pm 1$ \\
HBP/A13/DOX & 38.0 & $8 \pm 2$ \\
HBP/A8/DOX & 42.0 & $7 \pm 1$
\end{tabular}

aDetermined by NMR diffusion measurements using the Stokes-Einstein equation; ${ }^{b}$ $\emptyset_{\mathrm{M}}=1.3$, determined by GPC (MALLS)

\section{Tumour regression studies}

While in vitro experiments presented in our previous study suggested the potential for improved therapeutic response of the targeted nanomedicine compared with the polymer control, it is important to verify these findings in an in vivo model. In this case a tumour regression model was chosen to compare the efficacy of the nanomedicines that were targeted with different aptamers against HSP70 (A8 and A13) and the untargeted analogue. Figure 2 shows the relative tumour size of MDA-MB-468 human breast cancer xenografts in nude mice, indicating therapeutic efficacy of five doxorubicin containing formulations compared with a PBS control. The formulations included DOX conjugated to hyperbranched polymer (HBP/DOX), DOX conjugated to hyperbranched polymer and targeted with aptamer A13 (HBP/A13/DOX), DOX conjugated to hyperbranched polymer targeted with aptamer A8 (HBP/A8/DOX), free DOX co-injected with hyperbranched polymer targeted with aptamer A13 (HBP/A13, Free DOX) and free DOX. A DOX equivalent dose of $4.5 \mathrm{mg} / \mathrm{kg}$ was used in each case in order to assess the effect of using a nanocarrier to deliver the therapeutic, and also to determine whether the different peptides showed improved efficacy when used as targeting groups on the nanomedicine. All DOX containing formulations showed suppression of tumour growth when compared to administration of PBS (Figure 2A). In particular, both $\mathrm{HBP} / \mathrm{A} 8 / \mathrm{DOX}$ and HBP/A13/DOX showed statistically significant increased inhibition of tumour growth compared to either HBP/DOX or DOX alone $(p<0.003)$. This indicated that the aptamer displayed either a chemo-sensitizing effect when working together with doxorubicin to enhance accumulation of DOX in the tumour, or a combination of both mechanisms was at play. Furthermore, HBP/A13/DOX showed slightly higher effectiveness at suppressing tumour growth compared to $\mathrm{HBP} / \mathrm{A} 8 / \mathrm{DOX}$, which also matches previously reported results that show that the 13-amino acid peptide reproduces the HSP70-blocking chaperone and exhibits anti-tumour properties in isolation; this is especially evident in vivo. ${ }^{13}$ In addition, both DOX alone and the control experiment in which HBP/A13 was co-administered with free DOX, showed a similar ability to suppress tumour growth.

The effects of chemotherapeutic toxicity is evident in Figure 2B, where all DOX-conjugated polymer treatments (HBP/DOX, HBP/A13/DOX, $\mathrm{HBP} / \mathrm{A} 8 / \mathrm{DOX}$ ) show minimal adverse effects in the mice, where they exhibit no loss in weight during the therapeutic regime compared to the PBS control group. In contrast, the treatment group in which free DOX was administered in combination with HBP/A13 displayed statistically significant weight loss (compared to the saline control; $\mathrm{p}<0.01$ ), with $20 \%$ weight loss over the experimental timeframe. The reason for this apparent excessive toxicity compared to just free DOX is not known (free DOX treatment group showed far lower toxic effect compared to saline control), however it may be related to the reported sensitising effect of aptamer A13 where co-delivery with free DOX enhances this effect in functional organs (such as liver, kidney, heart etc.). ${ }^{19}$

Finally, histological images (hematoxylin and eosin (H\&E) stain; Figure 2C) of tumour sections excised from the different treatment groups showed that the number of dead cells in the tumours of mice treated with both HBP/A8/DOX and HBP/A13/DOX are larger compared to that of either HBP/DOX or free DOX. This suggests more of an efficacious therapeutic effect when the drugs are delivered using an aptamer-targeted nanomedicine, as opposed to systemic administration of free drug. 

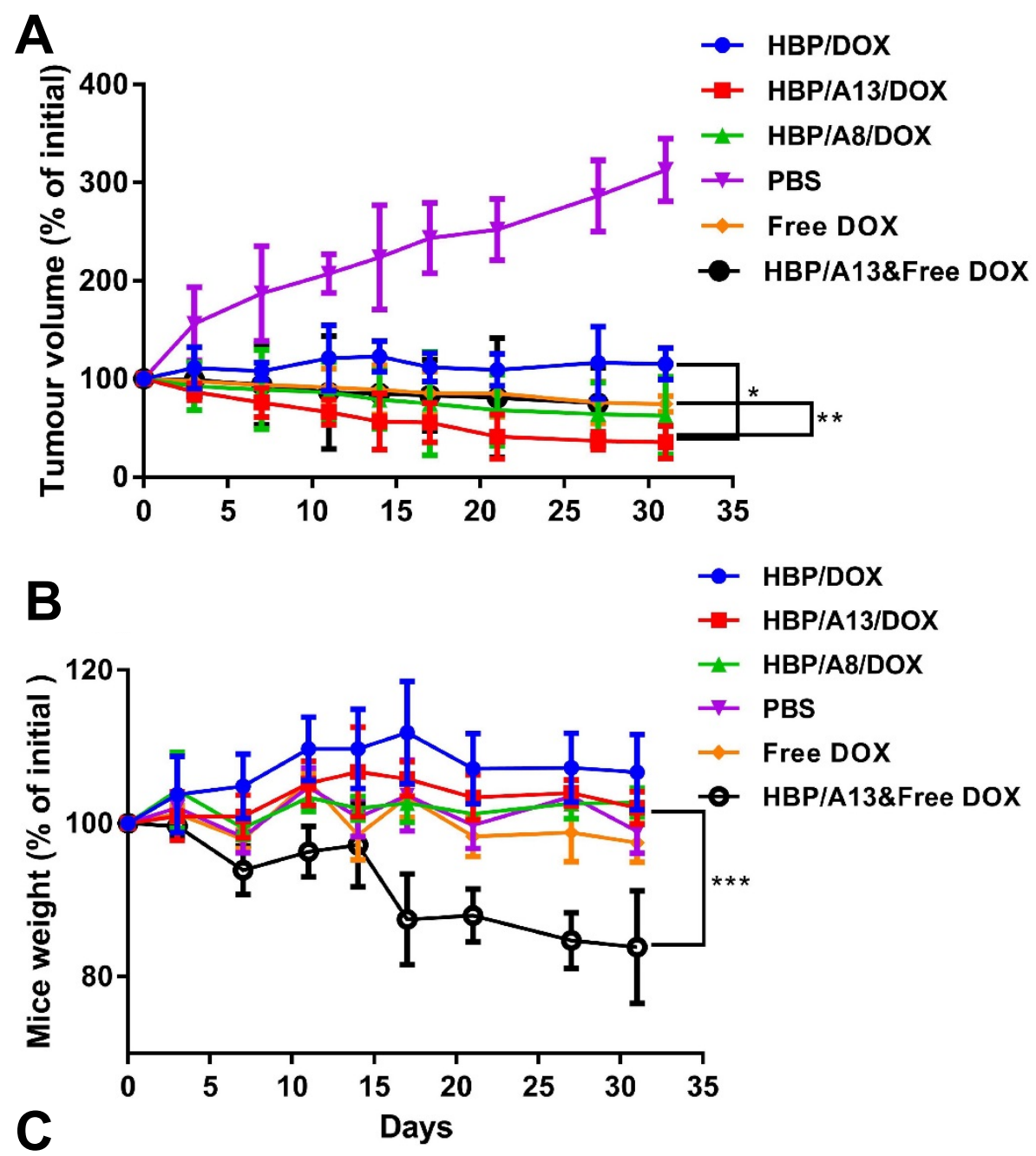

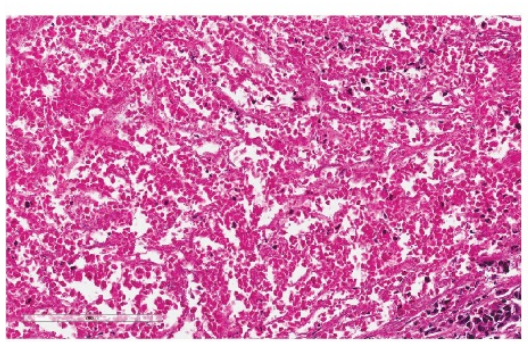

HBP/A8/DOX

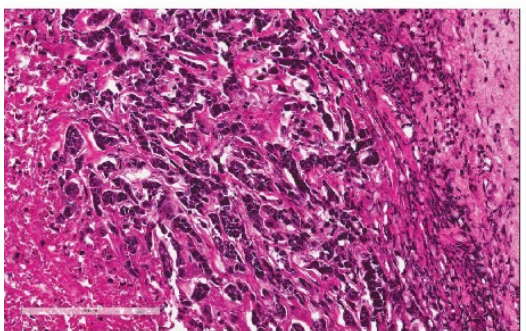

HBP/A13\& Free DOX

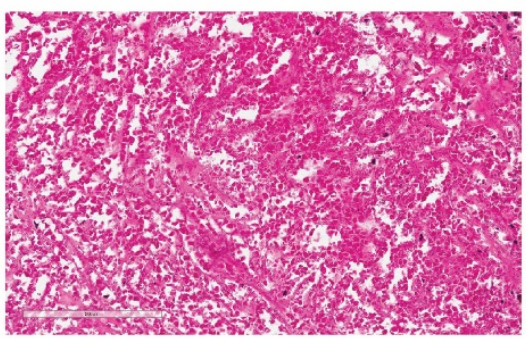

HBP/A13/DOX

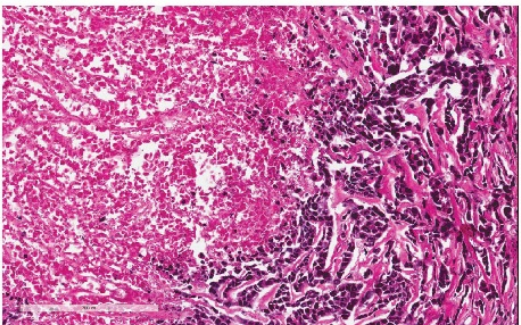

Free DOX

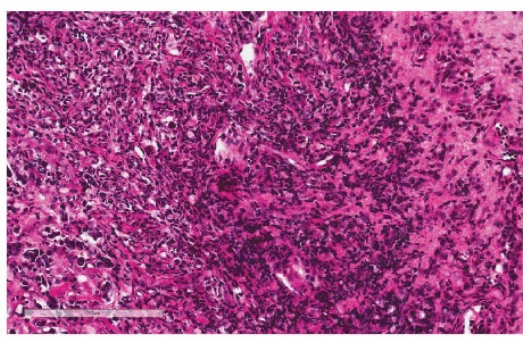

$\mathrm{HBP} / \mathrm{DOX}$

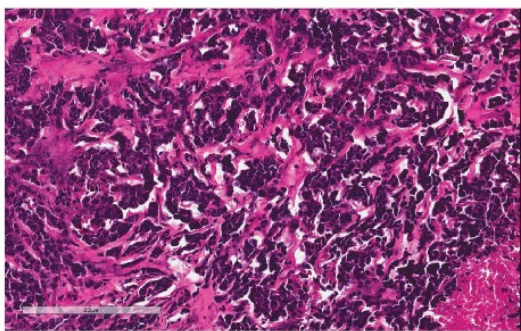

PBS

Figure 2 Tumour growth inhibition of s.c human breast MDA-MB-468 carcinoma xenografts in BALB/c nude mice. Mice were injected i.v. with 4.5 mg/kg DOX equivalent dose: Saline, free DOX, HBP/DOX, HBP/A8/DOX, HBP/A13/DOX, HBP/A13\&free DOX. (A) Tumour volume change and (B) body weight change ( $\mathrm{n}=3$ ) Values are the means and error bars the standard deviations $\left(n=3\right.$, S.D.) $\left({ }^{*} p<0.003,{ }^{*} p<0.004\right.$, $\left.{ }^{* * *} p<0.01\right)$. (C) Representative histological images (H\&E stained) of slices of MDA-MB-468 xenograft tumours two days after final treatment, scale bars: $200 \mu \mathrm{m}$. 

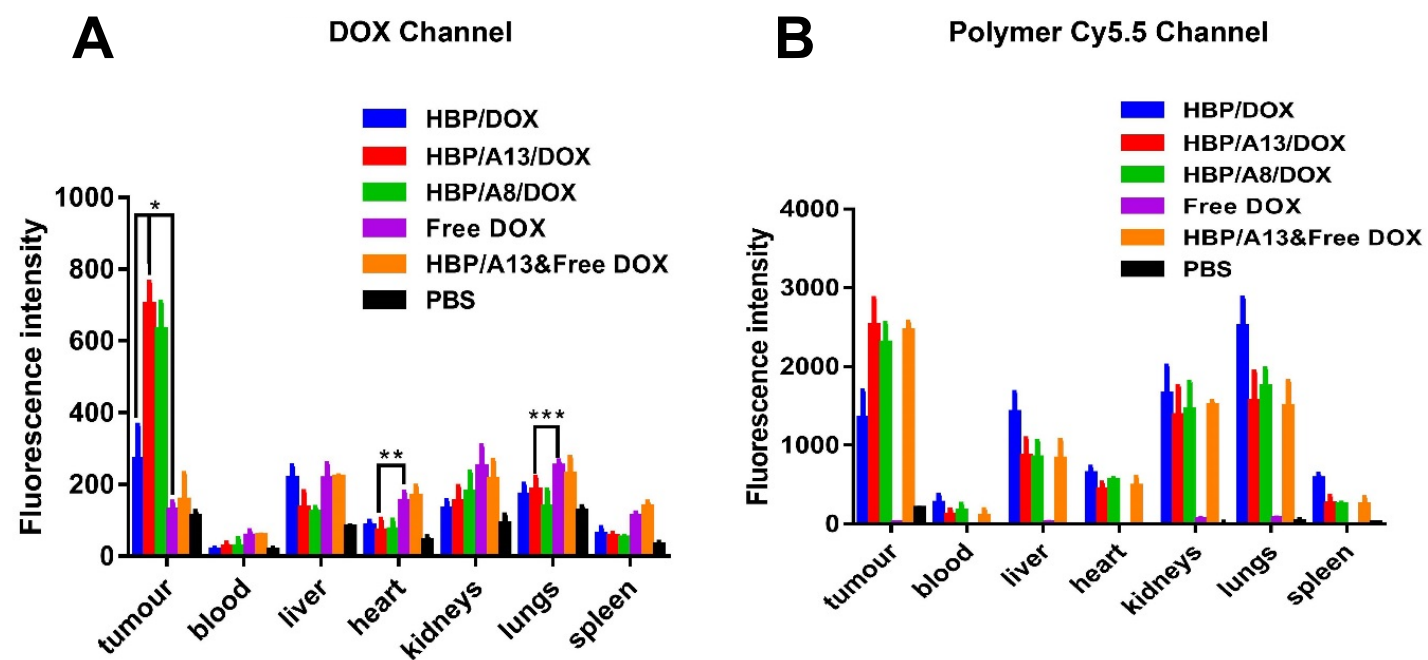

Figure 3 Quantitation of optical images of blood and tissues (tumour, liver, heart, spleen, lung and kidney) after i.v. administration (4.5 mg/kg DOX equivalent dose) of different drug formulations against s.c. human breast MDA-MA-468 carcinoma xenografts. Mice were sacrificed at $48 \mathrm{~h}$ after last treatment. (A) DOX fluorescence intensity level in blood and other organs. (B) Cy5.5 (polymeric carriers) fluorescence intensity level in blood and other organs. Values are the means \pm the standard deviations ( $\mathrm{n}=3$, S.D.) $\left({ }^{*} \mathrm{p}<0.0001,{ }^{* *} \mathrm{p}<0.05, * * * \mathrm{p}<0.2\right)$

\section{Ex vivo Biodistribution}

The relative distribution of the different components in the major organs of the mice were measured by ex vivo fluorescence imaging of excised organs at $48 \mathrm{hrs}$ post-injection of the final treatment. Relative fluorescence intensity of free DOX and DOX loaded HBPs in blood and different organs (tumour, liver, heart, kidneys, lungs and spleen) is shown in Figure 3.

Figure $3 \mathrm{~A}$ clearly shows that the mice treated with aptamer-targeted formulations (HBP/A8/DOX, HBP/A13/DOX) led to significantly increased DOX accumulation in the tumour at this time point compared to HBP/DOX ( 2.6-fold increase; p < 0.0001), HBP/A13 co-delivered with free DOX or free DOX alone ( 5-fold increase; $p<0.0001)$. Specifically, the accumulation of DOX in the tumours by HBP/A13/DOX was approximately 5.5 times higher compared to free DOX after 28 days treatment, and $\mathrm{HBP} / \mathrm{A} 8 / \mathrm{DOX}$ was at approximately 5 times higher levels. In the absence of any significant self-quenching of the fluorophores, these differences were attributed to enhanced accumulation of the polymers in the tumour tissue as a function of the aptamer binding to HSP70 on the tumour cells. It is also evident from the Cy5.5 channel (which is a measure of the nanocarrier accumulation) that there was no significant difference in HBP accumulation between the HBP/A8/DOX, $\mathrm{HBP} / \mathrm{A} 13 / \mathrm{DOX}$, in line with the DOX distribution. The differences in distribution between organs between polymer signal and DOX signal can most likely be attributed to the gradual clearance of polymer from the tumour environment over the time course of the therapy (while DOX most likely remains bound tightly to oligonucleotides within the nuclei of the cells as is often observed in vitro). Most importantly for downstream application of these nanomedicines, DOX concentration in the heart typically showed statistically-significant reduction in accumulation for the HBP/A8/DOX, HBP/A13/DOX groups compared to treatment groups when free DOX was administered (again, either as free DOX or as a combination therapy with $\mathrm{HBP} / \mathrm{A} 13$; $\mathrm{p}<0.05$ ); DOX is known to cause cardiotoxicity when administered alone.[2], [3]

\section{Intra-tumoral distribution of nanomedicine within xenografts}

To provide detailed information on the distribution of nanomedicine (and DOX) within the tumour microenvironment and to assess whether the diffusion of both nanocarrier and/or drug into tumour tissue affects efficacy, fluorescence imaging of tumour slices at the completion of the treatment study was undertaken. Figure 4 shows representative fluorescence images of slices of the xenografted tumours from each treatment group that highlight the perivascular distribution of the native fluorescence of doxorubicin (green), the polymeric carrier labelled with cyanine5.5 (red) and the cell nuclei that have been stained with DAPI (blue)) in relation to anti-CD31 stained blood vessels (orange). Looking first at the free DOX treatment group, the DOX channel shows a relatively weak signal, which is distributed uniformly throughout the slice. The HBP/DOX treatment group exhibits strong signals from the polymer carrier (red) that are predominantly 
co-localized or adjacent to tumour blood vessels (orange) and a weaker signal that is distributed more uniformly throughout the tumour. In contrast, the DOX signal (green) was distributed throughout the slice and not significantly co-localised around the blood vessels (yellow). The regions where there is a lack of co-registration (at least in intensity of signal) between the DOX and polymer signals demonstrate that drug release is occurring from the carriers and the higher DOX signal for the HPB/DOX compared to the free DOX treatment groups suggests that enhanced accumulation of nanomedicine (and drug) occurs in this xenograft model compared to the drug alone. The targeted therapeutics, HPB/A8/DOX and HBP/A13/DOX treatment groups exhibit similar distribution profiles to the HBP/DOX. Again, the regions that lack co-registration of the polymer and DOX signals show that drug release is occurring in the tumour environment. Finally, the HPB/A13 that was co-delivered with the free DOX treatment group shows co-localisation of the polymer with the blood vessels, and a weak uniform distribution of DOX, similar to that observed for the free DOX case.

In order to better analyse these images, intensity plots of fluorescence signal were generated across the tumour slice to investigate the relationship between fluorescence intensity of DOX and the polymeric carrier, and to understand how this relates to diffusional distance from blood vessels. The comparison between groups showed that both targeted HBP/A8/DOX and HBP/A13/DOX showed higher DOX distribution throughout the tumour mass (maximum intensity on the y-scale in supporting information Figure S2) compared to the other three untargeted groups (DOX, HBP/DOX and HBP/A13 co-delivered with free DOX) where weaker DOX signals were observed. Moreover, in these cases, the large carriers appear to have a significant proportion of the signal remaining very close to the vessel wall (only penetrating a few cells distances into the tumour tissue), while the smaller size of DOX released from the polymeric carriers appears to diffuse throughout

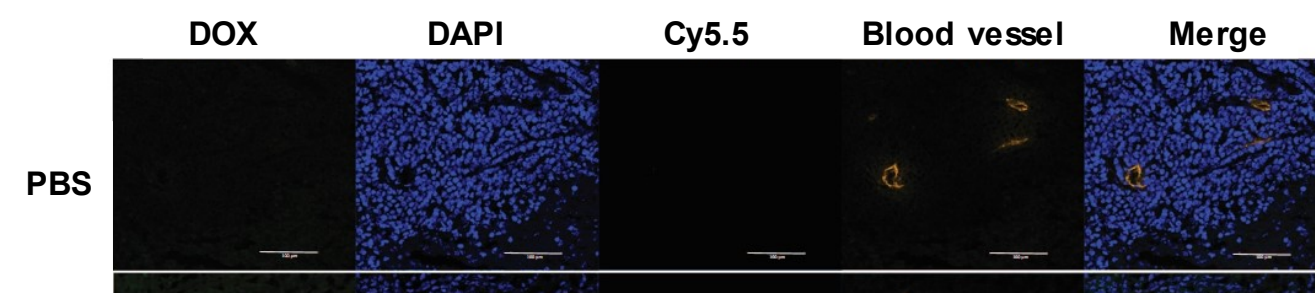

DOX

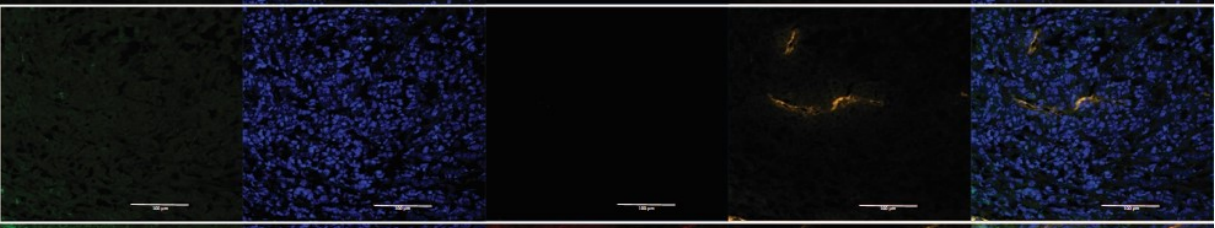

HBP/DOX

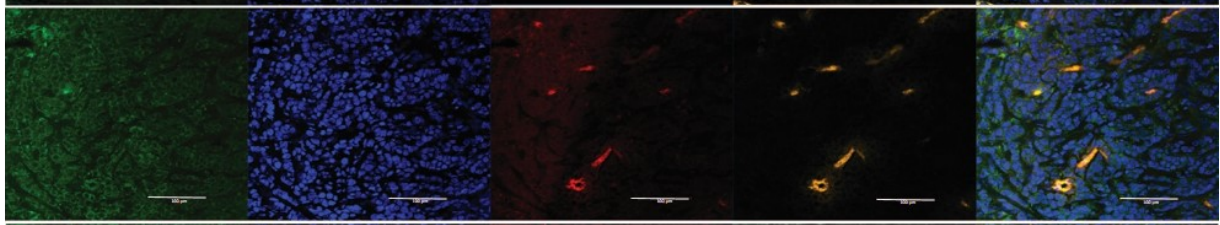

HBP/A8/DOX

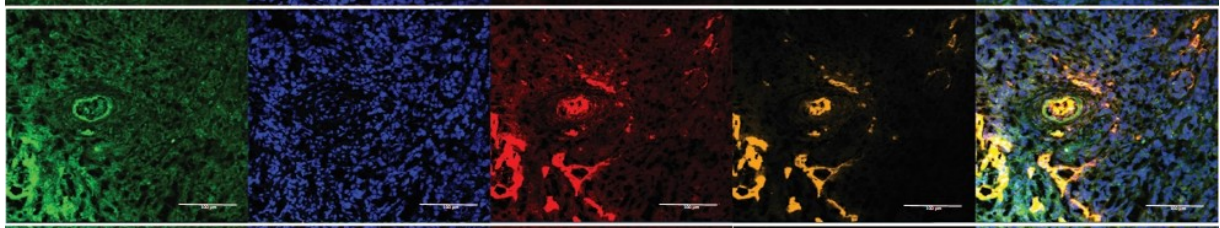

HBP/A13/DOX

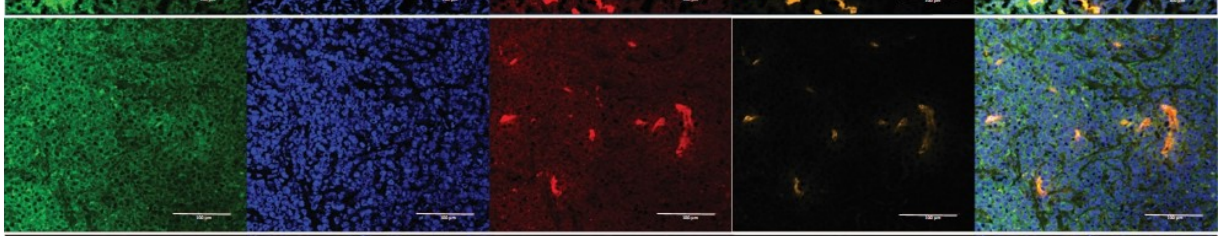

HBPIA13\&free DOX

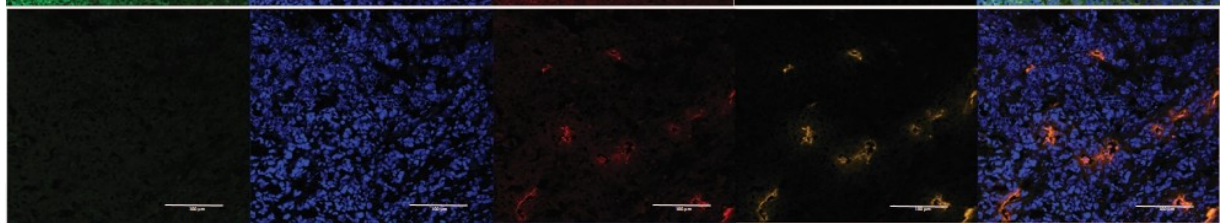

Figure 4 Distribution within tumour slices of doxorubicin (green) and polymer (red) in relation to tumour blood vessels (orange) and cell nuclei (blue). Scale bar $=100 \mu \mathrm{m}$ 
the tumour matrix. This is presumably due to the hydrophilic nature of DOX as well as its much smaller size compared to the nanomedicine, leading to a diffusion front of drug that penetrates well into the tumour microenvironment. This is probably most evident for the targeted systems using Aptamer 13, where there is significant accumulation within the region of the blood vessel. Nonetheless, the signal assigned to the nanomedicine (Cy-5.5) is still able to effectively distribute throughout the tumour to some extent in all treatment groups (albeit to a lesser extent than the DOX once it is released).

\section{In vivo MSOT imaging of targeted and untargeted nanomedicines in live animals}

MSOT is a relatively new technique that allows monitoring of both endogenous (e.g. haemoglobin) and exogenous probes.[29], [30] It has been reported that many tumour microenvironment properties such as tumour type, size and stage could affect the penetration of nanoparticles,[31] and that the effectiveness of chemotherapeutics could be influenced by their distribution through the tumour.[32] In order to better understand how the different treatments can distribute through the tumour microenvironment, MSOT was used to investigate how accumulation within the tissue was related to the vasculature in living animals. The strong vascular accumulation of the nanomedicines (independent of whether they were targeted or not), suggested that there was preferential accumulation close to blood vessels within the tumour. To better demonstrate the in vivo accumulation of $\mathrm{HBP} / \mathrm{A} 13 / \mathrm{DOX}$ compared to HBP/DOX, MSOT images were acquired of live mice during weeks 3 and 4 of the therapeutic regime to determine whether a noticeable change in polymer distribution could be observed which might give an indication on any improvements in targeting efficiency of the aptamer system. The images in Figure 5 show the long-term accumulation during weeks 3 and 4 of the Cy5.5-labelled HBP/DOX and HBP/A13/DOX (green) with respect to the endogenous signals of oxyand deoxyhemoglobin $\left(\mathrm{HbO}_{2}\right.$ (red) and $\mathrm{Hb}$ (blue), respectively [33]) within the tumour volumes. Note that DOX is undetectable by this technique owing to the low wavelength of absorption (absorption cutoff is $550 \mathrm{~nm}$ ) and the enhanced signal around the mouse image in the targeted experiment was due to artefacts whose origin has been previously reported.[34, 35] In both the HBP/DOX and HBP/A13/DOX, higher nanocarrier accumulation was observed in the tumour tissue compared to the PBS control, although a much higher signal was evident in the targeted nanomedicine. MSOT further allows in vivo quantification of signal accumulation and Figure 5C highlights an approximately 5-fold increase in accumulation of the targeted nanomedicine (HBP/A13/DOX) compared to the untargeted system (HBP/DOX). As was observed in the ex vivo analyses presented in Figures 4 and supporting information Figure S2, the localisation of the Cy5.5-labelled polymers was noted to have a vascular-dependent distribution, where the polymer appeared (at least on the resolution of this imaging modality) to be close to blood vessels or pools in the tumour (Figure 5A and $5 B)$ as a consequence of slow diffusion away from blood vessels into the tumour tissue.

\section{Conclusion and summary}

This report has demonstrated the effective inhibition of xenograft tumours in mice when treated with a number of therapeutic formulations. Both HBP/A8/DOX and HBP/A13/DOX showed significant inhibition of tumour growth, which supports the previous report that these aptamers act as antagonists for HSP70 in cancer cells. By utilising the specificity for HSP70, the aptamer targeted polymers can therefore provide a means to significantly enhance accumulation of nanotherapeutics within tumour tissue; in the case of this study, this led to significantly enhanced DOX levels in the tumour microenvironment. Free DOX showed some degree of effectiveness in leading to a regression in tumour volume, but histological analyses clearly show the greater efficiency in accumulation of therapeutics when using the targeted formulations compared to those that were untargeted. We propose that the use of the peptide aptamer to bind to HSP70 not only led to higher accumulation, but also longer retention at the site of the tumour presumably because of the ligand-receptor interaction.

There were no significant adverse health conditions observed in any of the treatment groups where doxorubicin was conjugated to polymer (HBP/DOX, HBP/A13/DOX and HBP/A8/DOX). This indicated that conjugation of DOX via a hydrazone bond was significantly stable in circulation such that systemic levels of the drug did not reach a level where toxicity was observed. Conversely, mice that were exposed to the combination treatment of $\mathrm{HBP} / \mathrm{A} 13$ with free DOX showed significant weight loss, perhaps due to the HBP/A13 conjugate acting as an antagonist for HSP70 that is active in cancer cells and also healthy cells. The ex vivo biodistribution result further validated the observation that A13 and A8 lead to almost the same level of tumour targeting efficacy. It was observed from the tumour regression study that HBP/A13/DOX showed slightly improved 
tumour inhibition compared to HBP/A8/DOX. This may be related to previous observations that A13 has improved chemosensitizing effects compared to A8,[19] and when combined with a chemotherapeutic drug this effect is exacerbated in a synergistic fashion. Nonetheless, both formulations showed effective treatment of the disease.

MSOT images of mice treated with aptamer targeted nanomaterials resulted in an enhanced uptake in the tumour volume compared to untargeted polymer. A combination of both ex vivo fluorescence imaging and MSOT imaging showed that the distribution of the HBP nanomedicines in solid tumours only occurred to a low extent. Contrastingly, the ex vivo analyses show that both high tumour accumulation and efficient intra-tumoural diffusion of DOX occurs for HBP/A8/DOX and HBP/A13/DOX. This implies that in terms of developing new nano therapeutics for delivery of DOX to solid tumours, the penetration of the nanomedicine may not be a critical factor. Rather, mechanisms for increasing tumour microenvironment accumulation, as well as efficient release of the DOX is more important for treating the tumours since the DOX appears to effectively diffuse independently into tumour tissue.

A
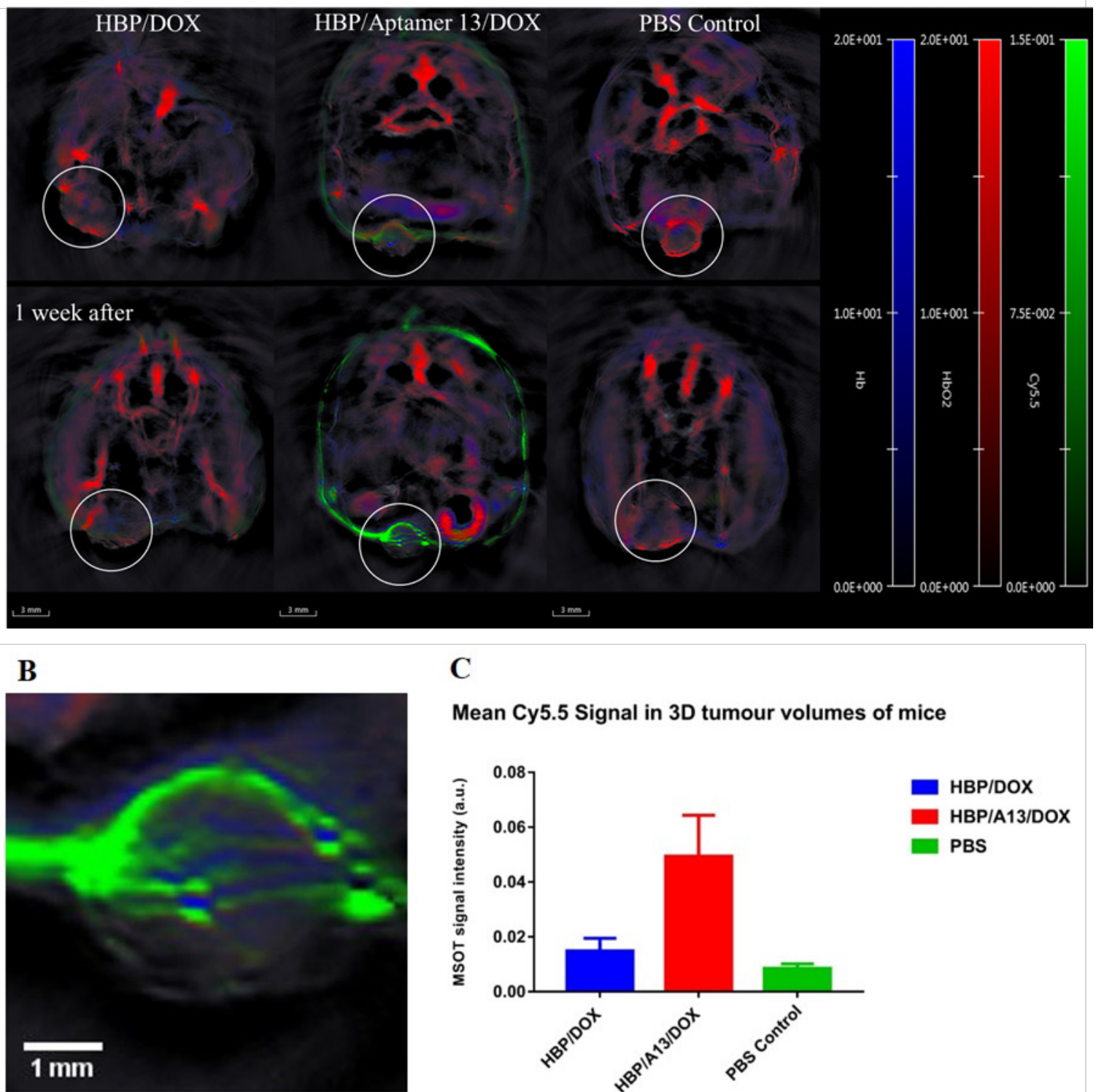

C

Mean Cy5.5 Signal in 3D tumour volumes of mice
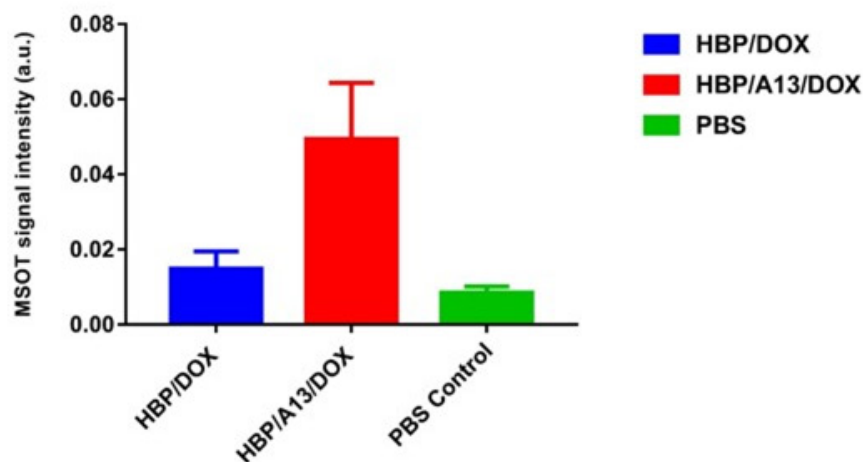

Figure 5 (A) Representative in vivo transverse MSOT images of mice at the largest cross-section point of each tumour (circled in white) in mice treated with HBP/DOX, HBP/A13/DOX and PBS during the $3^{\text {rd }}$ and $4^{\text {th }}$ weeks of treatment. Spectrally unmixed MSOT signal from accumulated Cy5.5 (green, 0-0.15 a.u.) can be seen with respect to blood vessels through spectral unmixing of signal from oxygenated (red, 0-20 a.u.) and deoxygenated haemoglobin (blue, 0-20 a.u.) overlayed on a single wavelength illumination at $845 \mathrm{~nm}$ (greyscale). All spectra used for signal unmixing can be found in the supporting information (Figure S4) A magnification of the tumour volume of the mouse treated with targeted polymer after 4 weeks is shown in B. (C) Comparison of the mean Cy5.5 signal in each tumour volume clearly shows the highest accumulation in the targeted HBP/A13/DOX mouse. Error bars indicate the variation in signal intensity between images acquired 1 week apart in a single mouse. 
In conclusion, these results demonstrate that both A8 and A13 significantly enhance the accumulation efficiency of HBP-based nanomedicines in vivo and offer new avenues for exploring nanomedicine treatments for cancer.

\section{Acknowledgements}

The authors acknowledge financial support from the Australian Research Council (LP150100703, CE140100036), the National Health and Medical Research Council (APP1099321, APP1148582) and the University of Queensland for a student scholarship (YZ). We acknowledge the use and support from the Australian National Fabrication Facility (Queensland Node) and the National Imaging Facility.

\section{Supplementary Material}

Supplementary figures.

http://www.ntno.org/v02p0360s1.pdf

\section{Competing Interests}

The authors have declared that no competing interest exists.

\section{References}

1. Jemal A, Bray F, Center MM, Ferlay J, Ward E, Forman D. Global cancer statistics. Ca-Cancer J Clin. 2011;61(2):69-90.

2. Chatterjee K, Zhang J, Honbo N, Karliner JS. Doxorubicin Cardiomyopathy. Cardiology. 2010; 115: 155-62

3. Shi Y, Moon M, Dawood S, McManus B, Liu PP. Mechanisms and management of doxorubicin cardiotoxicity. Herz. 2011; 36: 296-305.

4. Magiorakos AP, Srinivasan A, Carey RB, Carmeli Y, Falagas ME, Giske CG, et al. Multidrug-resistant, extensively drug-resistant and pandrug-resistant bacteria: an international expert proposal for interim standard definitions for acquired resistance. Clinical Microbiology and Infection. 2012; 18: 268-81.

5. Etheridge ML, Campbell SA, Erdman AG, Haynes CL, Wolf SM, McCullough J. The big picture on nanomedicine: the state of investigational and approved nanomedicine products. Nanomedicine: Nanotechnology, Biology and Medicine. 2013; 9: 1-14

6. Bobo D, Robinson KJ, Islam J, Thurecht KJ, Corrie SR. Nanoparticle-Based Medicines: A Review of FDA-Approved Materials and Clinical Trials to Date. Pharm Res. 2016; 33: 2373-87.

7. Lammers T, Aime S, Hennink WE, Storm G, Kiessling F. Theranostic Nanomedicine. Accounts of Chemical Research. 2011; 44: 1029-38.

8. Ahmed N, Fessi H, Elaissari A. Theranostic applications of nanoparticles in cancer. Drug Discov Today. 2012; 17: 928-34

9. Singh M, Harris-Birtill DC, Markar SR, Hanna GB, Elson DS. Application of gold nanoparticles for gastrointestinal cancer theranostics: A systematic review. Nanomedicine. 2015; 11: 2083-98.

10. Liu Y, Yang K, Cheng L, Zhu J, Ma X, Xu H, et al. PEGylated FePt@Fe2O3 core-shell magnetic nanoparticles: potential theranostic applications and in vivo toxicity studies. Nanomedicine. 2013; 9: 1077-88.

11. Yu T, Chan KW, Anonuevo A, Song X, Schuster BS, Chattopadhyay S, et al. Liposome-based mucus-penetrating particles (MPP) for mucosal theranostics: demonstration of diamagnetic chemical exchange saturation transfer (diaCEST) magnetic resonance imaging (MRI). Nanomedicine. 2015; 11: 401-5.

12. Pearce AK, Simpson JD, Fletcher NL, Houston ZH, Fuchs AV, Russell PJ, et al. Localised delivery of doxorubicin to prostate cancer cells through a PSMA-targeted hyperbranched polymer theranostic. Biomaterials. 2017; 141: 330-9.

13. Mayer MP, Bukau B. Hsp70 chaperones: Cellular functions and molecular mechanism. Cellular and Molecular Life Sciences. 2005; 62: 670-84.

14. Ciocca DR, Clark GM, Tandon AK, Fuqua SA, Welch WJ, McGuire WL. Heat shock protein hsp70 in patients with axillary lymph node-negative breast cancer: prognostic implications. J Natl Cancer Inst. 1993; 85: 570-4.

15. Hwang TS, Han HS, Choi HK, Lee YJ, Kim YJ, Han MY, et al. Differential, stage-dependent expression of Hsp70, Hsp110 and Bcl-2 in colorectal cancer. J Gastroenterol Hepatol. 2003; 18: 690-700.

16. Ricaniadis N, Kataki A, Agnantis N, Androulakis G, Karakousis CP. Long-term prognostic significance of HSP-70, c-myc and HLA-DR expression in patients with malignant melanoma. Eur J Surg Oncol. 2001; 27: 88-93.
17. Sherman MY, Gabai VL. Hsp70 in cancer: back to the future. Oncogene. 2015; 34: 4153-61.

18. Horibe T, Torisawa A, Kohno M, Kawakami K. Synergetic cytotoxic activity toward breast cancer cells enhanced by the combination of Antp-TPR hybrid peptide targeting Hsp90 and Hsp70-targeted peptide. BMC Cancer. 2014; 14: 615.

19. Rerole AL, Gobbo J, De Thonel A, Schmitt E, Pais de Barros JP, Hammann A, et al. Peptides and aptamers targeting HSP70: a novel approach for anticancer chemotherapy. Cancer Res. 2011; 71: 484-95.

20. Zhao Y, Houston ZH, Simpson JD, Chen L, Fletcher NL, Fuchs AV, et al. Using Peptide Aptamer Targeted Polymers as a Model Nanomedicine for Investigating Drug Distribution in Cancer Nanotheranostics. Molecular Pharmaceutics. 2017; 14: 3539-49.

21. Coles DJ, Rolfe BE, Boase NR, Veedu RN, Thurecht KJ. Aptamer-targeted hyperbranched polymers: towards greater specificity for tumours in vivo. Chem Commun (Camb). 2013; 49: 3836-8.

22. Rolfe BE, Blakey I, Squires O, Peng H, Boase NRB, Alexander $\mathrm{C}$, et al. Multimodal Polymer Nanoparticles with Combined 19F Magnetic Resonance and Optical Detection for Tunable, Targeted, Multimodal Imaging in Vivo. J Am Chem Soc. 2014; 136: 2413-9.

23. Pearce AK, Rolfe BE, Russell PJ, Tse BWC, Whittaker AK, Fuchs AV, et al. Development of a polymer theranostic for prostate cancer. Polymer Chemistry. 2014; 5: 6932-42.

24. Morscher S, Driessen $\mathrm{WH}$, Claussen J, Burton NC. Semi-quantitative Multispectral Optoacoustic Tomography (MSOT) for volumetric PK imaging of gastric emptying. Photoacoustics. 2014; 2: 103-10.

25. Neuschmelting V, Burton NC, Lockau H, Urich A, Harmsen S, Ntziachristos V, et al. Performance of a Multispectral Optoacoustic Tomography (MSOT) System equipped with 2D vs. 3D Handheld Probes for Potential Clinical Translation. Photoacoustics. 2016; 4: 1-10.

26. Yushkevich PA, Piven $J$, Hazlett $H C$, Smith $R G$, Ho $S$, Gee JC, et al User-guided 3D active contour segmentation of anatomical structures: significantly improved efficiency and reliability. Neuroimage. 2006; 31: 1116-28.

27. Tuteja A, Mackay ME, Narayanan S, Asokan S, Wong MS. Breakdown of the Continuum Stokes-Einstein Relation for Nanoparticle Diffusion. Nano Letters. 2007; 7: 1276-81.

28. Mackay ME Carmezini G. Manipulation of Hyperbranched Polymers' Conformation. Chemistry of Materials. 2002; 14: 819-25.

29. Gujrati V, Mishra A, Ntziachristos V. Molecular imaging probes for multi-spectral optoacoustic tomography. Chemical Communications. 2017; 53: 4653-72.

30. Ma R, Taruttis A, Ntziachristos V, Razansky D. Multispectral optoacoustic tomography (MSOT) scanner for whole-body small animal imaging. Opt Express. 2009; 17: 21414-26.

31. Perrault SD, Walkey C, Jennings T, Fischer HC, Chan WCW. Mediating Tumor Targeting Efficiency of Nanoparticles Through Design. Nano Letters. 2009; 9: 1909-15.

32. Steyger PS, Baban DF, Brereton M, Ulbrich K, Seymour LW. Intratumoural distribution as a determinant of tumour responsiveness to therapy using polymer-based macromolecular prodrugs. Journal of Controlled Release. 1996; 39: $35-46$.

33. Tomaszewski MR GI, O'Connor JP, Abeyakoon O, Parker GJ, Williams KJ, Gilbert FJ, Bohndiek SE. Oxygen Enhanced Optoacoustic Tomography (OE-OT) Reveals Vascular Dynamics in Murine Models of Prostate Cancer. Theranostics. 2017; 7: 2900-13.

34. Tzoumas S, Ntziachristos V. Spectral unmixing techniques for optoacoustic imaging of tissue pathophysiology. Philosophical Transactions of the Royal Society A: Mathematical, Physical and Engineering Sciences. 2017; 375.

35. Quli F, Kai C, Zhen Y, Ruiping Z, Min Y, Xiang H, et al. Perylene-Diimide-Based Nanoparticles as Highly Efficient Photoacoustic Agents for Deep Brain Tumor Imaging in Living Mice. Advanced Materials. 2015; 27 : 843-7. 